\title{
MONDIALISATION, MÉTROPOLISATION ET CHANGEMENT URBAIN EN AFRIQUE DU SUD
}

\author{
Philippe Gervais-Lambony
}

Depuis une dizaine d'années, deux phénomènes majeurs ont touché les villes d'Afrique du Sud : la mondialisation économique et la démocratisation politique. La métropolisation est alors devenue pour les autorités sud-africaines la clé de l'adaptation à ces bouleversements, comme le montre l'exemple de l'East Rand, grande région urbaine et industrielle située à l'Est de Johannesburg.

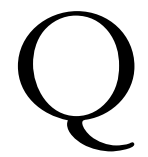
ue se passe-t-il aujourd'hui dans les grandes agglomérations du monde? La question est ambitieuse, mais ne doit-elle pas être la toile de fond de toute recherche sur des cas particuliers urbains? Le contexte actuel de la recherche urbaine, quelle que soit la discipline concernée, conduit à cette question. Qu'entend-on dire et que lit-on, en effet? Que la fin du $20^{\mathrm{e}}$ siècle, depuis les années 1970, s'est caractérisée par des changements de grande ampleur dans le domaine urbain, notamment de nature économique. Que ces changements ont eu des conséquences si considérables sur les espaces urbains et les sociétés citadines qu'il faut peut-être parler d'une nouvelle révolution urbaine. La littérature scientifique, notamment anglo-saxonne, nous dit clairement que nous sommes entrés dans l'ère du "post ": postmoderne, post-fordiste, post-industriel, voire post-ville ${ }^{1} \ldots$ Nous serions donc dans un après.

On peut ne pas vouloir regarder si loin. Premièrement l'opposition entre un avant et un après est forcément simplificatrice : les traces du passé sont là, des fonctionnements anciens coexistent avec des fonctionnements nouveaux. Ni l'après ni l'avant n'existent donc absolument, nous sommes dans un présent qui est mélange d'hier et de demain, notre objet d'étude est sans doute le plus souvent la façon dont se combinent et interagissent l'ancien et le nouveau. Deuxièmement, tous les espaces urbanisés du monde ne changent pas en même temps ni au même rythme, toutes les villes du monde ne partent pas sur les mêmes bases et un même phénomène mondial peut avoir des effets contradictoires sur deux espaces différents. Les études comparatives, de plus en plus nombreuses ${ }^{2}$, montrent les différences entre les espaces urbanisés en Amérique du Nord, en Europe, dans les pays dits du Sud, dans les anciens pays à économie socialiste ; audelà ces études démontrent des différences profondes à l'intérieur même de ces ensembles ; enfin, les études monographiques soulignent les irréductibles spécificités de chaque cas particulier. Cela ne signifie pas que les espaces urbanisés ne sont pas soumis aux mêmes forces, mais que ces forces ont des effets divers dans l'espace et décalés dans le temps. Cela ne signifie pas non plus qu'aucun des changements ur-

1. Thierry Paquot, "Études urbaines ou "sciences de la ville et des territoires" ", in T. Paquot, M. Lussault, S. BodyGendrot (dir.), La ville et l'urbain l'état des savoirs, Paris, La Découverte, 2000, p. 5-17. Edward W. Soja, Postmetropolis. Critical studies of cities and regions, Oxford, Blackwell Publishers, 2000

2. Cf. notamment Françoise Dureau et al. (dir.), Métropoles en mouvement, Paris, Anthropos-IRD, 2000. 
bains contemporains n'est commun à toutes les agglomérations du monde. Il ne faut donc pas renoncer à la question générale : quels changements affectent les espaces urbains actuellement? Pourquoi et comment?

\section{○ LES BOULEVERSEMENTS URBAINS ACTUELS}

Au pourquoi, il y a une réponse rapide : ce sont les effets de la mondialisation qui provoquent les changements urbains. C'est recourir à un mot attrape-tout, certes, mais personne ne peut nier qu'il a son efficacité pour décrire la compression du monde, la conscience accrue de son unicité, l'intensification des flux (de capitaux, de produits, de personnes), un changement dans le rôle des États (mais certainement pas la disparition de leur rôle), la concentration à l'échelle mondiale des pôles de commandement économiques et la mise en relation les uns avec les autres de tous les lieux du monde. Le social, le culturel, le politique et bien sûr l'économique s'en trouvent affectés. Cependant, ce qui est nouveau ce n'est pas la mondialisation en soi mais son intensité et son universalité, nouveauté partielle donc, mais qui suffit à changer le monde.

Quels sont les effets de la mondialisation sur les villes ? Le changement de la relation ville/industrie est classiquement avancé ${ }^{1}$. Pour le dire très grossièrement, c'est le processus de désindustrialisation de la ville qui semble dominer l'histoire urbaine contemporaine, donnant naissance à des formes et des fonctionnements urbains très différents de ceux issus de la révolution urbaine entamée au $18^{\mathrm{e}}$ siècle en Europe et qui avait débouché, au $20^{\text {e }}$ siècle, sur la ville industrielle dite fordiste. Ceci se vérifie évidemment d'abord dans les grandes villes des pays industrialisés. Dans les

1. S. Sassens, La ville globale. New York, Londres, Tokyo, Paris, Descartes, 1996 (traduction française de The Global City, 1991). A. J. Scott, New industrial spaces, flexible production. Organisation and regional development in North America and Western Europe, Londres, Pion, 1988. villes du reste du monde, il y a parfois un effet contradictoire qui est l'industrialisation par des processus de délocalisation de la production. Mais cette industrialisation se fait le plus souvent à côté des espaces urbanisés. Il n'est donc pas question de dire que les villes ne sont plus les lieux de l'industrie, en général, mais que le rapport ville/industrie a changé et que les activités industrielles se sont déplacées et, dans beaucoup de cas, ont changé de forme.

Admettons donc que mondialisation et transformation du rapport ville/industrie justifient que l'on pense que la nature des espaces urbanisés et des sociétés citadines change. Du côté du changement de l'espace (la distinction entre espace et société est ici purement rhétorique, l'un ne va pas sans l'autre dans la réalité), on relèvera l'extension des espaces urbanisés, leur étalement spatial et leur croît démographique, et surtout leur caractère désormais polycentrique $^{2}$. La métropole industrielle n'est plus ce qu'elle était. Les espaces métropolitains, les conurbations, les aires urbanisées se multiplient. Du côté du changement social, on rencontre un autre terme générique, celui de fragmentation. La ville polycentrique connaît des divisions socio-spatiales nouvelles, plus fortes sans doute, et surtout la société urbaine, à l'échelle de l'agglomération, ne fait plus tout ; le référent global et commun à la ville est comme perdu. L'affaiblissement de la classe moyenne, la disparition de la classe ouvrière telle qu'on la connaissait, l'exclusion socio-économique d'une partie des citadins et au contraire l'extrême enrichissement d'autres, donnent naissance à une société urbaine brisée, fragmentée. Et ceci a des conséquences sensibles sur le plan politique et gestionnaire : dans beaucoup de grandes agglomérations du monde, il n'y a pas ou plus de niveau politique commun, mais juxtaposition de territoires municipaux et en conséquence parcellisation aussi de la ges-

2. R. Kloosterman, S. Musterd, "The polycentric urban region : towards a research agenda ", Urban Studies, 2001, vol. 38,4 , p. 623-633. 
tion urbaine. Une abondante littérature portant sur les changements urbains actuels décrit cette tendance générale à l'éclatement des organismes urbains ${ }^{1}$. Les réalités auxquelles ces auteurs font référence sont diverses : fractionnement spatial de la ville, disparition du lien social citadin, division économique de la ville, séparation politique de territoires, replis communautaires, renfermement des plus riches dans des cités fermées, mise à l'écart spatiale des plus pauvres... Mais la plupart jugent utile la notion de fragmentation pour décrire non pas forcément le passage à un nouvel ordre urbain mais au moins l'émergence de nouveaux types d'espaces dans les villes et de relations différentes entre ces espaces ${ }^{2}$ différents de la ségrégation traditionnelle.

La notion de fragmentation peut donc servir de résumé des effets socio-spatiaux de la mondialisation sur l'urbain. On parle d'une hypothèse de changement global (spatial, social, politique, économique) et l'on débouche sur la question de l'identité de la ville et des citadins. À quelle échelle faut-il aujourd'hui chercher une identité citadine ? Ce qui revient à se demander si la ville existe encore en tant que collusion entre un espace et une société fonctionnant comme un tout. D'où l'emploi courant, pour désigner les effets actuels de la mondialisation sur les villes, du terme de métropolisation. Ce terme a plusieurs dimensions. Le sens étymologique de ville mère, ville d'origine d'une civilisation urbaine, n'est sans doute plus le principal. L'emploi contemporain du terme indique d'autres choses : l'étalement de la ville à une échelle nouvelle, la concentration des pouvoirs politiques et économiques dans certaines villes (les métropoles) ou encore un changement dans le fonctionnement

1. Cf. Françoise Navez-Bouchanine (dir.), La Fragmentation en question. Des villes entre fragmentation spatiale et fragmentation sociale?, Paris, L'Harmattan, 2002.

2. Peter Marcuse et Ronald Van Kempen (dir.), Globalizing cities. A new spatial order?, Oxford, Blackwell Publishers, 2000. politique de la ville, soit parce qu'il devient cogestion d'un espace par plusieurs entités politiques (des municipalités), soit parce que sont mises en place des structures métropolitaines de gouvernement des villes. Dans le même temps, pourtant, les changements urbains contemporains ont induit un changement de fonctionnement des agglomérations, au moins dans certaines parties du monde, conduisant certains auteurs à rejeter le terme de métropole comme décrivant une réalité passée, à savoir la métropole industrielle de la période moderne (ou fordiste), et la distinguant de la postmétropolis $^{3}$. Le temps des métropoles modernes serait donc passé. C'était une ville industrielle en partie centrée sur un centreville unique concentrant les fonctions de commandement économique, entourée de banlieues diverses en Europe, de vastes suburbs en Amérique du Nord, de quartiers populaires, souvent hors de la légalité, dans les pays dits pauvres, c'était un ensemble inégalitaire et ségrégué mais au moins fondé sur un fonctionnement économique commun. Si la ville actuelle se fragmente, elle s'oppose à la métropole ainsi entendue, les territoires changent d'échelle, au lieu de devenir plus vastes avec l'étalement de l'agglomération, ils se réduisent à des enclaves juxtaposées. Et en même temps, les citadins métropolitains ont aussi des espaces territoriaux de plus en plus complexes et discontinus (et différents de ceux de leurs voisins).

Traiter de la métropolisation dans un cas national particulier oblige donc à un choix terminologique. Le plus simple, pour commencer, est de fonder ce choix sur les réalités locales. Que signifie métropolisation dans le contexte sud-africain?

\section{O MONDIALISATION ET MÉTROPOLISATION À LA MODE SUD-AFRICAINE}

L'Afrique du Sud a plusieurs villes mères. Le Cap est celle des populations

3. Edward W. Soja, Postmetropolis..., op. cit. 
d'origine européenne : point d'arrivée des colonisateurs européens au milieu du $17^{\mathrm{e}}$ siècle, la ville est à l'origine d'une civilisation urbaine originale fondée sur le racisme et l'esclavage. Kimberley, première grande cité minière (l'exploitation du diamant y a commencé dans les années 1860) dont le mode de gestion de la maind'œuvre africaine (à laquelle fut imposé le système du travail migrant) a servi de modèle au $20^{\text {e }}$ siècle pourrait être considérée comme la ville mère de l'apartheid. Mais les cités africaines dans la région sont aussi mères, Ulundi, capitale du royaume zoulou, Zimbabwe, au-delà des frontières actuelles de l'Afrique du Sud, coeur d'un des royaumes du Monomotapa, etc.

Au sens politique du terme, l'Afrique du Sud a aussi plusieurs métropoles : Pretoria, siège du gouvernement et du pouvoir exécutif, Le Cap, siège du Parlement et Bloemfontein, siège du pouvoir judiciaire puisque s'y trouve la Cour suprême. À un autre niveau territorial, neuf villes sont capitales administratives et politiques des neuf provinces d'Afrique du Sud, métropoles régionales donc.

Au sens économique, c'est la ville de Johannesburg qui est la vraie métropole sud-africaine. Fondée à la fin du $19^{\text {e }}$ siècle sur les gisements aurifères du Witwatersrand (les plus importants du monde à l'époque), Johannesburg fut d'emblée une métropole économique internationale. Siège des grands groupes financiers sud-africains, disposant du premier aéroport d'Afrique australe, Johannesburg est aussi la ville la plus peuplée du pays et se trouve au cœur d'une région urbaine de 8 millions d'habitants, le Gauteng, qui concentre le tiers des emplois nationaux et produit plus du tiers du PIB sud-africain.

Mais, si l'on réfléchit au sens sud-africain du terme métropolisation et à ses relations avec la phase actuelle de la mondialisation, il est un autre angle d'approche possible. Ce qui s'est passé au début des années 1990 dans ce pays est une révolution politique de grande ampleur : la fin et le dé- mantèlement du régime de l'apartheid, remplacé par un système politique démocratique et idéologiquement fondamentalement différent. C'est sur cet aspect que s'est focalisée jusqu'à récemment la recherche urbaine sur l'Afrique du Sud : comment les villes sud-africaines passentelles d'un système politique à l'autre ${ }^{1}$ ? Mais au moment même où l'Afrique du Sud changeait de régime politique, elle réintégrait une communauté internationale en pleine mondialisation. C'est là la grande particularité du cas sud-africain, qui se reflète directement dans les changements urbains : comment gérer simultanément démocratisation, rattrapage social et spatial et mondialisation? L'outil choisi par les nouvelles autorités sud-africaines pour résoudre cette contradiction est l'établissement d'autorités métropolitaines dans les grandes villes du pays.

Depuis décembre 2000, date des deuxièmes élections locales post-apartheid en Afrique du Sud, dans le contexte d'une redéfinition des gouvernements locaux à l'échelle nationale, les six plus grandes agglomérations sud-africaines (Pretoria, Durban, Port Elisabeth, Johannesburg, le Cap et l'East Rand) sont devenues des " autorités métropolitaines " par fusion des différentes entités administratives qui les composaient jusque-là ${ }^{2}$. Dans la foulée, en 2001, trois de ces nouvelles autorités ont adopté de nouveaux noms, africains en

1. Claire Benit, La fragmentation urbaine à Johannesburg, thèse de doctorat en géographie, université de Poitiers, 2001. Philippe Gervais-Lambony, "Nouvelle Afrique du Sud, nouvelles frontières, nouvelles identités ? ", L'Espace géographique, 1999, 2, p. 99-109. Philippe Guillaume, Johannesburg. Géographies de l'exclusion, Paris, Karthala, 2001.

2. Les autorités métropolitaines sont un des trois types possibles d'autorités locales définies par la constitution de 1996 et surtout par le Livre Blanc sur le Gouvernement Local (Ministry for Provincial Affairs and Constitutional Development, 1998). Les Metros ont l'exclusivité du pouvoir exécutif et réglementaire sur leur juridiction. Les Districts englobent plusieurs municipalités avec lesquelles ils partagent les pouvoirs. Les Municipalities sont englobées dans des Districts et partagent avec eux pouvoirs et responsabilités. Pour définir les nouveaux types d'autorités locales et leurs limites, le gouvernement a établi une commission, le Demarcation Board (régie par le Municipal Demarcation Act de 1998) mise en place à partir du premier février 1999. 
général : Tswane Metro pour Pretoria, Nelson Mandela Metro pour Port Elisabeth et Ekurhuleni Metro pour l'East Rand.

Les critères officiels pour justifier la création (ou pas) d'une Metro avaient été définis par le Municipal Structure Act de 1998. Ce statut devait être accordé aux aires urbanisées polycentriques, économiquement importantes, diversifiées, liées socialement et économiquement par un fonctionnement commun. Cinq des six agglomérations devenues des Metros remplissaient sans conteste ces conditions. Seul le cas de l'East Rand posait problème car le fonctionnement commun de cet ensemble n'était pas démontré et les oppositions locales à la métropolisation étaient nombreuses. Nous nous appuierons sur cet exemple dans ce qui suit pour apporter des éléments de réponse à une question d'ordre général : la "métropolisation " politique (mise en place d'autorités métropolitaines) permet-elle de limiter les effets négatifs de la mondialisation (notamment la fragmentation des organismes urbains) ? Le cas de l'East Rand est intéressant parce qu'extrême : les autorités cherchent à faire d'un espace naguère particulièrement divisé politiquement, économiquement et socialement, un seul ensemble métropolitain. L'East Rand, plus de 2 millions d'habitants, est une région urbaine composée, selon les délimitations, de six à neuf municipalités (carte 1). Les inégalités socio-spatiales sont très fortes, opposant des secteurs racialement et économiquement contrastés. De plus, l'East Rand est spatialement très discontinu, faisant alterner zones inhabitées, zones de fortes densités, zones de faibles densités, zones d'activités économiques. La question posée est donc aussi interne à la métropole : constitue-t-elle une entité ? Qu'est-ce qui unit les territoires à l'intérieur de cet ensemble? Pour régler la question de l'autorité métropolitaine de l'East Rand, il était finalement nécessaire de répondre à des questions de fond sur la ville, puisque la loi disait assez clairement que les Metros devaient être des aggloméra- tions "faisant ville " c'est-à-dire ayant non seulement un fonctionnement commun mais aussi une identité commune ${ }^{1}$.

\section{○ CYCLES ÉCONOMIQUES ET HÉRITAGES DE L'APARTHEID DANS L'EAST RAND}

Il est nécessaire de dire brièvement l'histoire de la mise en place de la structure spatiale de l'East Rand pour comprendre ce qui fait son unité mais aussi sa disparité interne. La région urbaine de l'East Rand, incluse dans le Witwatersrand, est née à la fin du $19^{e}$ siècle et s'est développée sur une base économique minière. Toutes les villes de cet ensemble (à l'exception d'Alberton et Kempton Park) ont été des centres miniers. Le second cycle économique régional, qui remonte à la seconde guerre mondiale ${ }^{2}$ fut industriel : toutes les villes de l'East Rand, à des degrés divers, sont devenues alors des centres industriels (métallurgie et chimie surtout). Les années 1950 et 1960 furent une période de croissance économique rapide de la région, et aussi de croissance démographique, surtout par l'afflux d'une population noire ouvrière employée dans les industries. C'est à cette époque que la région entra dans une ère fordiste de production de masse et devint une métropole industrielle (mais déjà polycentrique). De fait, chaque municipalité se rêvait alors métropole, en même temps que l'ensemble restait sous la domination économique de Johannesburg. Cette période fut aussi celle de la mise en œuvre de politiques urbaines à l'échelle régionale visant à gérer la main-d'œuvre

1. Si l'on admet, avec Augustin Berque (Du geste à la cité. Formes urbaines et lien social au Japon, Paris, Gallimard, 1993) que la ville est à la fois une forme (matérialité de la ville) et une substance (c'est-à-dire les hommes et les relations entre les hommes) qui coïncident pour donner un " sujet collectif ", c'est-à-dire que " la ville matérielle et ses habitants se co-influencent en une sorte de sujet collectif ", c'est la question de l'existence d'un tel sujet qui est posée ici.

2. Phillip Bonner, "African urbanisation on the Rand between the 1930s and 1960s : its social character and political consequences ", Journal of Southern African Studies, 1995, vol 21, 1, p. 115-129. 
africaine et son logement. À la fin des années 1950 et dans les années 1960, les autorités de l'apartheid imposèrent un vaste programme de réaménagement régional : tous les anciens quartiers noirs (les locations) furent rasés (comme ailleurs en Afrique du Sud) pour être remplacés par des quartiers en général périphériques, les townships. Ces ensembles de logements publics locatifs ont été planifiés à l'échelle de la région East Rand ${ }^{1}$ et répartis en quatre grands blocs de quartiers (carte 1). Cependant, malgré cette politique régionale, chaque municipalité conservait son propre quartier africain ; pour y résider, il fallait avoir un emploi dans la municipalité. On observe là la naissance d'une tension jamais disparue entre le pouvoir central qui tentait de traiter l'East Rand comme un seul ensemble et les municipalités qui résistaient à cette tendance. C'est en quelque sorte un conflit entre une vision métropolitaine de la région et une vision municipale. Si on lit cette histoire en continuité depuis les années 1950, on s'aperçoit que ce n'est qu'en 2000 que le pouvoir central l'a emporté en imposant la constitution d'une seule autorité métropolitaine. Entre-temps, les soubresauts ont été nombreux.

Dans les années 1980 la crise économique frappa sévèrement l'East Rand. Le cycle minier s'achevait avec la fermeture de la plupart des mines d'or, le cycle industriel fordiste débutait son déclin avec les premières fermetures d'usines d'industries lourdes. Ces deux cycles étaient certes relayés par le développement d'activités d'industrie de pointe, d'industries légères et surtout du secteur tertiaire, mais le nombre d'emplois était considérablement

1. Paul Hendler, "Living in apartheid's shadow. Residential planning for Africans in the PWV region 1970-1990 ", Urban Forum, 1992, 3-2, p. 39-80. Alan Mabin, "Conceptualising, making and governing the Witwatersrand ", communication présentée devant l'Africa's Urban Past Conference, SOAS, Londres, 1996. Mohamed Noor Nieftagodien, The Making of Apartheid in Springs : group areas and forced removals, Master thesis, University of the Witwatersrand, 1995. réduit et les espaces de ces nouvelles activités n'étaient ni les anciennes grandes zones industrielles, ni les anciens centrevilles, mais des zones situées surtout près de l'aéroport international de Johannesburg (Kempton Park) et au Nord de la région. Ceci laissait une structure spatiale héritée de l'apartheid, certes, et aussi, voire surtout, de cycles économiques anciens, tout à fait inadaptée.

La réponse des autorités à cette crise fut une fragmentation politique plus forte encore avec la création, à partir de 1982, d'autorités municipales spécifiques aux townships, les Black Local Authorities. Dans le même temps, chaque ville continuait d'avoir sa propre politique économique, en compétition avec ses voisines ${ }^{2}$.

Si l'on relit l'histoire de la région à la lumière de la question de la métropolisation on observe donc que :

- la métropolisation économique de type fordiste, combinée à la politique raciste de l'apartheid, a donné naissance à une forme urbaine particulière, spatialement éclatée, mais bel et bien au service du développement industriel ;

- les résistances municipales à la métropolisation ont bloqué une métropolisation politique complète ;

- depuis les années 1980, la crise de l'économie de type fordiste et le passage à ce qui semble bien devoir être qualifié de post-fordiste a laissé la région avec une structure spatiale en décalage ;

- la tension entre métropolisation politique imposée par le haut et résistance municipale s'est cependant maintenue après la fin de l'apartheid.

Ce dernier point est essentiel. Il prouve que la métropolisation politique n'est pas strictement une conséquence de la mondialisation mais aussi l'objet d'un enjeu de pouvoir entre pouvoir central et pouvoirs locaux. Ce conflit latent explique qu'il ait fallu attendre six ans après la fin de l'apar-

2. Ceci ne fut pas atténué par la mise en place en 1987 d'une autorité métropolitaine (Regional Service Council) bien peu puissante. 
theid (1994) pour que soit effectivement mise en place une autorité métropolitaine dans l'East Rand. Cette période a été celle d'un affrontement souvent direct entre les anciennes municipalités et le pouvoir central. Comme ailleurs en Afrique du Sud ${ }^{1}$, le découpage administratif de l'East Rand entre 1994 et 2000 a été le résultat de négociations ${ }^{2}$. Des instances locales de discussion sur les choix municipaux ont été mises en place. Y participaient les anciennes autorités municipales ${ }^{3}$, les partis politiques, les civics ${ }^{4}$, des représentants de la société civile et des milieux économiques. Dans les grandes aires urbaines, il s'agissait de faire un choix entre la mise en place de simples municipalités (réunissant un township et une ville blanche) ou d'une autorité métropolitaine englobant plusieurs anciennes municipalités. Au moment d'entrer dans ces négociations, la situation économique et politique dans l'East Rand était loin d'être bonne. Les BLA étaient en faillite, les townships plongés dans la violence, les municipalités blanches étaient pour la plupart très endettées, enfin les activités économiques traditionnelles de l'East Rand poursuivaient leur déclin.

Des négociations sortit un East Rand divisé. Sous la pression des municipalités les plus riches (qui craignaient la redistribution financière à l'échelle métropolitaine), les territoires municipaux furent reformés sur des bases anciennes. En réponse, les autorités provinciales du Gauteng imposè-

1. Robert Cameron (dir.), Democratisation of South African local government, a tale of three cities, Pretoria, J. L. Van Schaik, 1999.

2. Ivor Chipkin, "Les ambiguiités du gouvernement local en Afrique du Sud. Les cas de l'East Rand ", in P. GervaisLambony et al. (dir.), La question urbaine en Afrique australe, Paris, Karthala, 1999, p. 77-88.

3. L'East Rand était divisé en 19 autorités municipales (neuf anciennes BLA et dix municipalités "blanches "). De plus, couvrant presque l'ensemble de l'aire concernée, le East Rand Regional Service Council (ERRSC) participait aux négociations.

4. Associations qui se sont développées à partir du début des années 1980 pour défendre des intérêts locaux mais qui sont devenues des instances de représentation locale essentielles (voire les seules) pendant la crise des années 1980. Le mouvement des civics est partenaire de l'alliance politique actuellement au pouvoir en Afrique du Sud. rent la mise en place d'une instance de coordination régionale, le Eastern Gauteng Regional Service Council. Mais cette autorité avait juridiction sur un vaste territoire qui englobait aussi de vastes régions rurales (jusqu'au Nord de Pretoria) : les municipalités de l'East Rand se trouvaient devoir verser une partie de leurs revenus à une autorité qui les redistribuerait dans des zones rurales éloignées. La période 19942000 fut finalement marquée par le retour à la logique des municipalités, ce qui s'explique par une convergence d'intérêts : les élus blancs des ex-municipalités blanches avaient peur de perdre tout pouvoir dans une vaste autorité métropolitaine, les fonctionnaires municipaux craignaient une refonte globale de l'administration, les autorités provinciales pensaient conserver plus de pouvoir face à de petites municipalités que face à une seule autorité. Mais ce fut aussi l'occasion de voir combien les discours identitaires territoriaux à l'échelle municipale étaient efficaces puisque les opinions publiques locales, blanche comme noire, semblaient bien elles aussi affirmer leur attachement à leur ville. Ce fut un échec pour la conception métropolitaine de la région. Celle-ci fut finalement imposée, mais par le haut, en 2000. Tous les acteurs économiques et politiques de l'East Rand prétendaient être d'accord pour une planification intégrée mais ils n'en avaient pas la même définition. Du côté des autorités municipales, quand on ne rejetait pas tout à fait l'idée métropolitaine, on imaginait une simple coordination régionale, du côté du Demarcation Board et du gouvernement, on pensait à une réelle centralisation et à l'abolition des municipalités. Le gouvernement, sur avis d'experts, a tranché pour une autorité métropolitaine forte, divisée en 88 circonscriptions élisant 175 conseillers (99 sont des élus ANC) ${ }^{5}$

5. Le système est un peu plus complexe : un conseiller est élu par chaque circonscription électorale (ward) à la majorité, 87 autres sont élus à la proportionnelle sur des listes présentées par les partis politiques de façon à assurer une meilleure représentation des petits partis. 
mais politiquement dirigée par le maire et son comité exécutif de 10 membres.

Pourquoi le pouvoir central a-t-il pu en 2000 faire ce qu'il n'était pas parvenu à réaliser en 1994 ? Le contexte politique et économique de l'année 2000 l'explique. Cette année-là, l'ANC remporta pour la seconde fois les élections législatives, Nelson Mandela prit sa retraite et Thabo Mbeki lui succéda. La priorité du gouvernement était devenue la redéfinition du fonctionnement de l'État. Sur le plan économique, le Reconstruction and Development Program qui devait financer le rattrapage social de façon centralisée avait été remplacé dès 1997 par un programme au contraire nettement libéral, le Growth, Employement and Reconstruction Program. Dans les grandes villes, le temps n'était donc plus ni à la négociation politique avec les minorités, ni au renforcement du pouvoir central pour permettre la redistribution mais au contraire à la réforme libérale, la privatisation des services et la rationalisation... Le gouvernement central ne cherche donc plus tant aujourd'hui à se donner les moyens de contrôler les autorités locales qu'à en faire des agents de développement économique néo-libéraux, tout en conservant le pouvoir à l'ANC. D'où l'attrait pour la métropolisation : pour la première fois l'État sudafricain n'a plus intérêt au contrôle fort du pouvoir local qui était nécessaire au régime d'apartheid (pour gérer la question "indigène " et contrôler les flux de maind'œuvre) et aussi à l'ANC tant qu'il cherchait à mettre en œuvre un plan de redistribution économique sous la houlette de l'État.

\section{O MÉTROPOLISATION ET FRAGMENTATION TERRITORIALE}

Dans le cas particulier de l'East Rand, le discours officiel pour justifier la métropolisation était pluriel. Cette réforme était présentée à la fois comme devant permettre une plus grande efficacité et favo- riser la redistribution économique. Il devait donc s'agir d'aider la région à répondre à deux défis de la mondialisation : la nécessité d'être économiquement compétitive à l'échelle mondiale, l'obligation de résister à la tendance à la fragmentation urbaine dans un contexte de désindustrialisation. En d'autres termes, l'objectif de la réforme était la création d'un ensemble urbain fonctionnellement et socialement cohérent.

Que l'East Rand soit un ensemble fonctionnel n'est pas démontré. Certes les flux sont importants d'une municipalité à l'autre, qu'il s'agisse de flux de travail quotidiens, de flux résidentiels ou d'investissements. Mais la région est tiraillée entre deux autres échelles territoriales. Vers le haut, l'East Rand appartient au Witwatersrand et ses liens avec Johannesburg son considérables $^{1}$; de la même façon l'East Rand appartient au Gauteng : on a donc affaire à des ensembles urbains polycentriques et emboîtés, avec toutes les difficultés d'analyse que cela pose. Vers le bas, les sousensembles territoriaux sont aussi divers, les inégalités entre municipalités très fortes et les disparités internes plus encore. Surtout, les identités locales sont très affirmées et les identités des anciennes municipalités ont été nettement renforcées durant la période transitoire (1994-2000) ${ }^{2}$. Les identités territoriales locales sont aussi renforcées par le fait que la fin de l'apartheid a rendu très lisibles les impacts de la mondialisation économique sur la région. Le déclin des activités industrielles en est le principal élément. Actuellement l'Ekhuruleni Metro-

1. Certains courants d'aménageurs et de politiques arguaient d'ailleurs des liens très forts entre l'East Rand et le reste du Witwatersrand pour dire que c'est cet ensemble-là qui aurait du devenir " métropole".

2. Durant ces six années les municipalités ont procédé à un travail de redéfinition identitaire important par le biais de campagnes d'information, de réunions sur le terrain, de mise en place de nouveau symboles et logos municipaux dans le but de fonder leur identité "post-apartheid "(cf. P. Gervais-Lambony, De la citadinité au territoire. Itinéraire via quatre villes africaines, mémoire d'habilitation à diriger des recherches, université de Paris 10-Nanterre, 2001 et " Les enjeux d'une politique de redéfinition territoriale. La création de l'aire métropolitaine d'Ekurhuleni ", Autre Part, 21, 2002, p. 27-40.). 


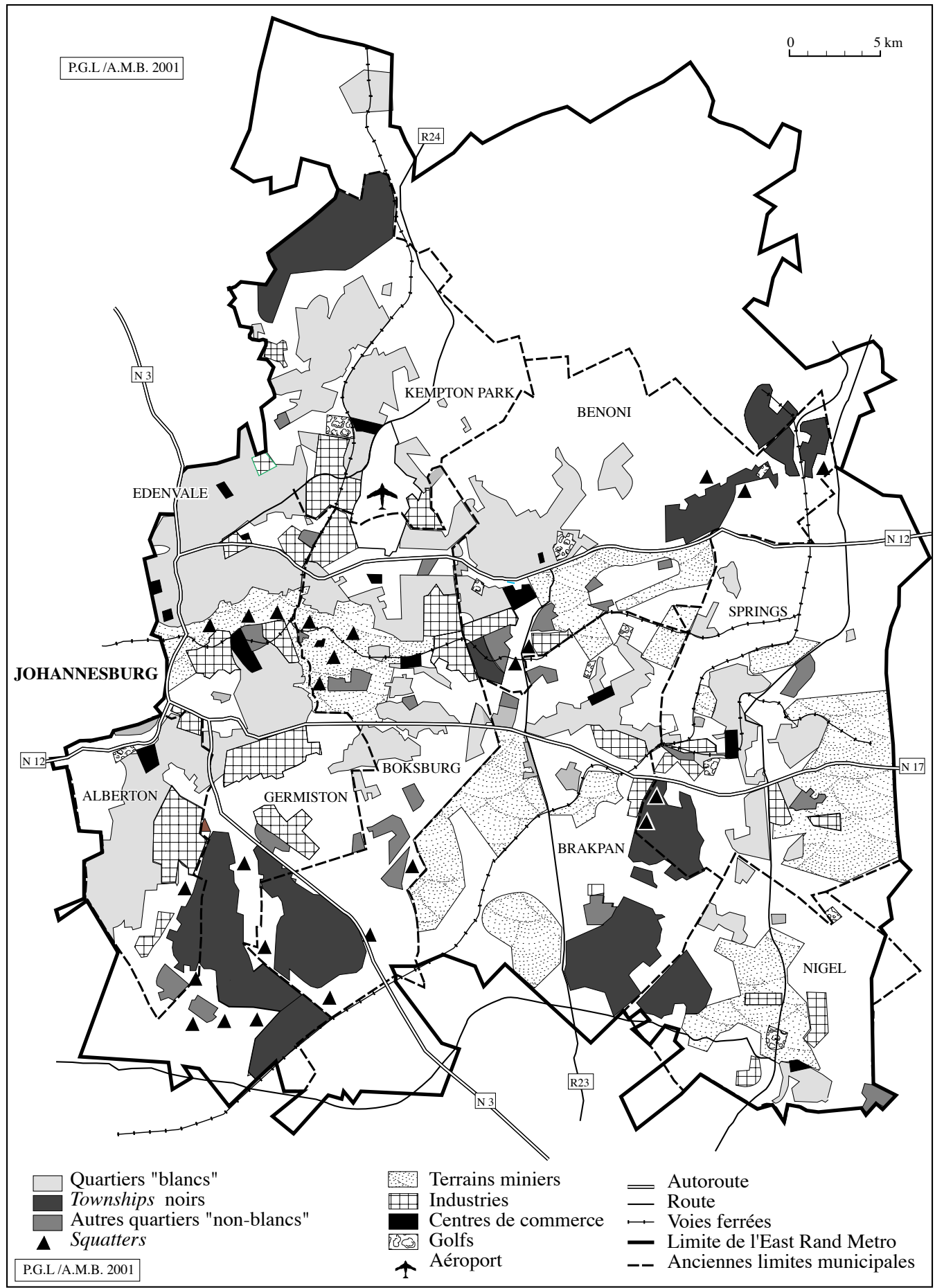

Carte 1. Occupation du sol dans l'East Rand. 


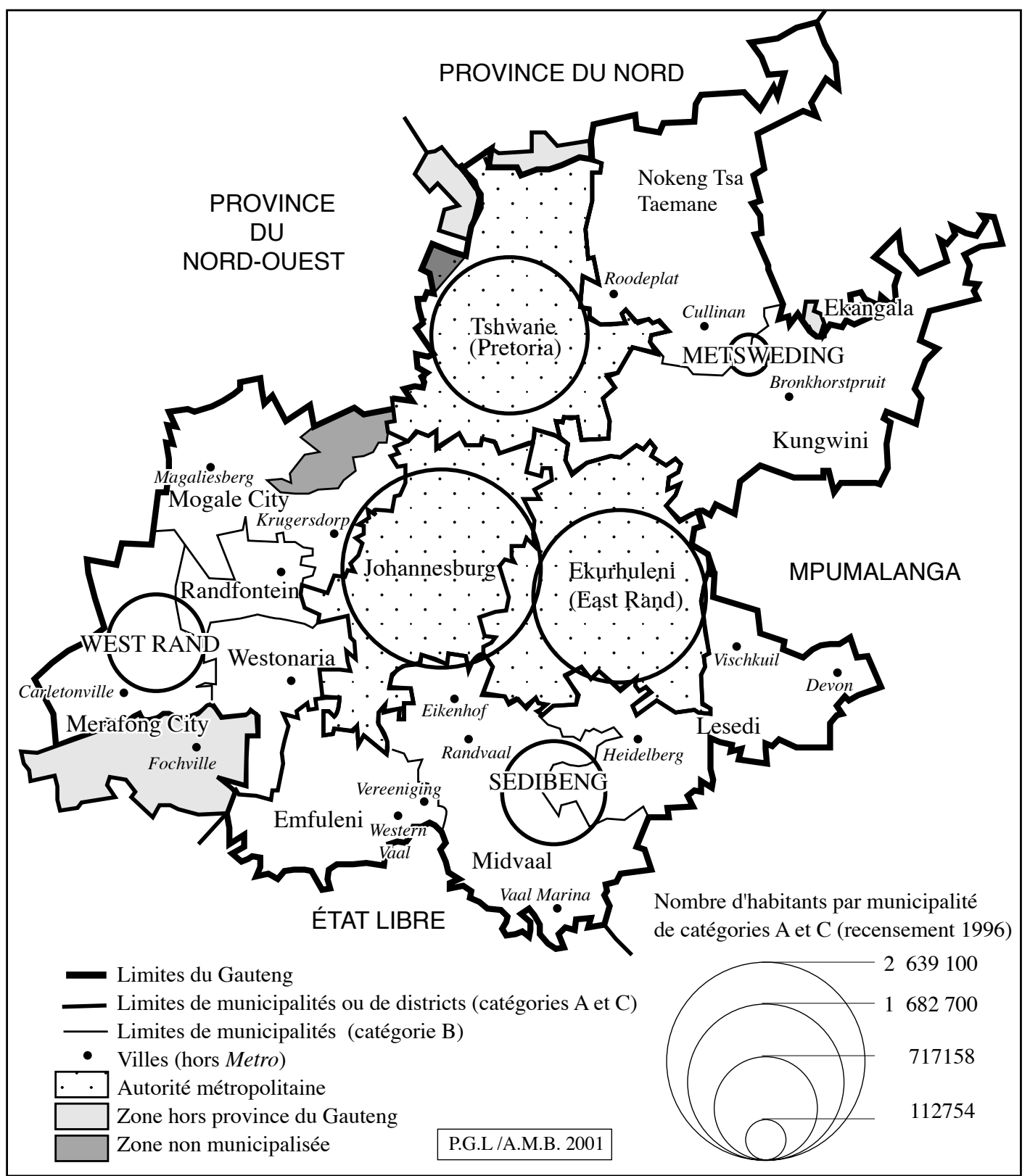

Carte 2. Les autorités locales dans le Gauteng depuis 2000

politan Area compte plus de 30 \% de chômeurs dans sa population active. Cette situation a provoqué l'exclusion de l'économie régionale de pans entiers de la population et d'espaces. Dans le même temps, les taux élevés de criminalité et les représentations largement fondées sur la peur provoquent le renfermement des classes aisées et moyennes. Aujourd'hui l'essentiel des constructions nouvelles 
dans les suburbs se fait sous la forme de lotissements fermés ; la ville riche se barricade, s'enferme derrière des systèmes d'alarme et des protections vendues par les compagnies privées de sécurité. Le déclin continu des anciens centres-villes au profit de vastes centres commerciaux de banlieue ne contribue pas au maintien de l'unité sociale de l'agglomération : les situations de co-présence entre les différents groupes sociaux sont de plus en plus rares. Non seulement les grands ensembles racialement définis restent en place, mais la fin de la ségrégation raciale légale semble bien renforcer la ségrégation sociale existante. Premièrement, les cas de mélanges raciaux sont extrêmement mineurs. Certes, une élite politique et d'affaires se déplace vers les banlieues naguère réservées aux Blancs, mais le phénomène n'est pas quantitativement important. Deuxièmement, dans le même temps, les contrastes sociospatiaux du côté de l'ancienne ville noire s'accentuent $^{1}$. Des townships part une partie de l'élite, et même ceux qui ne les quittent pas tendent à utiliser des services d'autres parties de la ville, notamment les écoles. À l'inverse, les plus pauvres sont actuellement logés dans de nouveaux quartiers périphériques, encore plus éloignés des centres que les anciens townships.

La politique de métropolisation de l'East Rand devrait aller dans un sens opposé à cette tendance à la fragmentation, mais ce pari est loin d'être gagné. D'abord la situation actuelle de transition entre 19 autorités locales et une seule entité métropolitaine provoque des dysfonctionnements administratifs graves. La plupart des citadins sont, à tout le moins, mal informés des changements d'échelles de leur représentation démocratique. Mais, au-delà, la mé-

1. Cf. à ce propos J. Beall, O. Crankshaw, S. Parnell, Uniting a divided city. Governance and social exclusion in Johannesburg, Londres, Earthscan, 2002 et Watson V., "L'accès au logement en Afrique du Sud ", in P. GervaisLambony et al. (dir.), La question urbaine en Afrique australe, Paris, Karthala, 1999, p. 227-241. tropolisation politique crée aussi de nouvelles frontières internes : celles des trois nouvelles Service Delivery Region (division de la métropole en trois secteurs pour la distribution et la gestion des services urbains), celles des nouveaux wards électoraux (niveau de représentation politique local et d'organisation des partis politiques) et celles aussi des projets de développement économiques, centrés sur certaines zones d'activités. Ceci se surimpose aux anciennes limites municipales, aux limites des quartiers, etc. Enfin, le poids de l'administration provinciale du Gauteng se fait sentir sur la métropole, surtout parce que l'essentiel des projets de développements est financé par des fonds régionaux (la province reçoit ses financements par subventions de l'État, elle est donc beaucoup plus riche que la Metro qui fonctionne sur ses propres revenus); la province du Gauteng fonctionne en fait comme une super-autorité métropolitaine handicapant par le haut toute réelle identification territoriale métropolitaine.

Les arguments pour la métropolisation politique étaient bons : remplacer par une planification urbaine régionale l'ancienne compétition entre municipalités ; faire table rase des divisions spatiales internes aux anciennes municipalités héritées de l'apartheid, rendre l'ensemble de l'agglomération compétitive au plan international. Moins affiché mais important, il y avait aussi un argument politique : un seul ensemble métropolitain permettait d'assurer mieux la prééminence politique de l'ANC et d'empêcher le maintien de bastions conservateurs tout en donnant le pouvoir à un maire désigné par le parti. Sur ce dernier point le succès est complet. Peu affiché aussi, il y avait derrière la métropolisation le souci de rationalisation économique: privatisation des services, simplification de la bureaucratie locale (et à terme sans doute réduction de personnel). 
Concluons sur l'ambiguité de la situation sud-africaine bien illustrée par le cas de l'East Rand. Subissant de plein fouet les effets de la désindustrialisation, comme une agglomération de pays du Nord, la ville sud-africaine n'a pas eu le temps de profiter de la fin du système d'apartheid : est-ce un voyage de Charybde en Scylla? La fin de la métropole industrielle est aussi la naissance de la métropole politico-administrative qui semble au moins autant favoriser la fragmentation spatiale que la limiter. Mais pouvait-on imaginer que la réforme des structures politiques pouvait aller contre la tendance lourde à la désindustrialisation/ fragmentation de l'agglomération qui a même traversé la rupture pourtant considérable qu'a été la fin du régime d'apartheid?

La métropolisation administrative estelle le bon outil pour unifier des espaces urbains fragmentés par la mondialisation ou favorise-t-elle au contraire la fragmentation? Pour en juger en Afrique du Sud, il faudrait tenir compte aussi de l'échelle temporelle : le changement économique, à partir d'une base industrielle, remonte aux années 1980, la métropolisation politique a à peine trois ans. Mais il est clair qu'il n'y a pas de lien mécanique entre unification politico-administrative et unification socioéconomique.

Philippe Gervais-Lambony est maître de conférences à l'université de Paris 10-Nanterre, Directeur du laboratoire Géotropiques, membre de l'Institut Universitaire de France, spécialiste de Géographie sociale sur les terrains urbains africains plus particulièrement d'Afrique australe. 